\title{
Study on the gas-liquid annular vortex flow for liquid unloading of gas well
}

\author{
Shuzhe Shi*, Xiaodong Wu, Guoqing Han, and Ziyao Zhong \\ College of Petroleum Engineering, China University of Petroleum, 18 Fuxue Road, Changping, Beijing 102249, China
}

Received: 30 May 2019 / Accepted: 19 September 2019

\begin{abstract}
Vortex tool is a new technique for the liquid unloading in gas wells. But it lacks a mathematical model to describe and predict the effect of vortex tools. In the present work, according to the axial, radial and circumferential momentum balance of the gas phase and liquid phase, the governing equations of vortex flow model have been established. Then thickness of liquid film and gas and liquid vortex flow intensity as well as the pressure drop gradient can be calculated. The calculation results and the previous experiments indicate that the pressure drop of the gas-liquid flow can be reduced by $5 \% \sim 25 \%$ with the vortex tool, and the vortex flow model has an average relative difference of $6.01 \%$. The model results show that there are two mechanisms for reducing the pressure drop under the vortex flow condition. In addition, the research results show that vortex tools with bigger helical angle will lead to higher vortex flow intensity. The decay rate of vortex flow intensity decreases along the pipe as liquid velocity increases and the vortex flow working distance can be calculated by the vortex intensity gradient and initial vortex flow intensity.
\end{abstract}

\section{Introduction}

Liquid loading is one of the significant issues needed to be overcome in gas-well production. When liquid loading occurs, gas production will be blocked from gas wells. Therefore, U.S. and China adopt vortex tools in liquid-loading gas wells in succession. Mingaleeva (2002) found that the minimum pressure loss can be obtained when the gasliquid fluid moves upward in vortex flow regime. Based on this theory, the downhole vortex tool in gas well was designed to decrease the pressure drop along the wellbore. The vortex tool transforms the common gas-liquid flow into swirling annulus flow with a certain stability. As a result, gas well production is increased and liquid carrying capacity is improved. Alekseenko et al. (1999) proposed a solution to symmetrical vortex accounting for the helical shape of vortex, and he conducted experiments on helical vortex that were carried out in a vertical hydrodynamical vortex pipe. Ali et al. (2005) first carried out an experimental study on vortex tools. They pointed out that pressure drop and critical gas velocity were reduced in vortex flow. Facciolo et al. (2007) used new measurements in a vortex pipe flow and came up with the axial average velocity distribution. Hein (2007) introduced the field test of vortex tools conducted by the U.S. Department of Energy from 2002 to

\footnotetext{
* Corresponding author: 20161312052@student.cup.edu.cn
}

2006. Milliken (2008) installed several Vortex Flow surface tools of different sizes in liquid gathering systems, he found that the Vortex Flow surface tool created two separated flows inside the stratified flow. Surendra et al. (2009) carried out a numerical simulation study on gas-liquid two phase flow process with the vortex tool. Singh et al. (2016) applied the vortex technology to gas lift with two cases. Field experiments also conducted on liquid loading wells simultaneously. The results indicated that the downhole vortex tool provided artificial lift optimization. Subsequently, a large number of field tests of vortex tools were gradually conducted in China ( $\mathrm{Du}, 2015$; Yang et al., 2012; Zhang et al., 2012; Zhu et al., 2013). In addition, experiments and theoretical researches on vortex tools were also gradually carried out in China. Wu et al. (2016) analyzed forces of liquid droplets in swirling flow based on two-phase fluid dynamics theories, and optimized the helical angle of vortex tools. Liu and Sun (2017) studied on the vortex tool with numerical method and conducted laboratory test and downhole test on geometrical parameters of the vortex tool. They found the preferred helical angles are $50^{\circ}$ and $55^{\circ}$. Zhou et al. (2018) established a critical liquid film model of gas wells under vortex flow conditions based on experimental data. Shi et al. (2018) simulated the gas-liquid flow process through the vortex tool under different flow regimes with Fluent software. Zhang et al. (2018) also used CFD method to simulate the flow process under different vortex tools, and analyzed the influence of 
number and distribution of vortex blades on liquid carrying performance.

Like other downhole liquid-unloading tools, the vortex tool cannot provide energy for gas-liquid two phase flow, but it can increase the efficiency of formation energy by improving flow conditions. However, extra energy losses can be increased with downhole tools at times. The vortex flow has a certain effective working distance that can be reflected by the stability of spiral rising liquid ring. The working distance will decrease as the angular velocity drops. Finally, the vortex flow disappears and returns to the original flow state.

Based on previous field experiments, it is found that the vortex tool has a certain application condition and an optimal operating condition. The unloading function of vortex tools can be well developed through reasonable wells choosing and vortex tools designing. Until now, there are no theoretical models for the tool operating characteristics and vortex flow characteristics. Due to the insufficient researches on the unloading mechanism of vortex tools, which limits the further applications of the vortex tools. The present work mainly focused on building a theoretical vortex flow model which is based on the two-phase flow theory and experiments were also conducted to validate the vortex model.

\section{Theoretical vortex flow model}

When the gas-liquid mixture flows through the vortex tools, liquid converges on the surface of vortex channel. The liquid ring will appear as shown in Figure 1, it is formed by the water which is from the liquid ring due to gravitation. Eventually, the gravitation of liquid ring will be equal to the gas drag force. Flow patterns are the basics of

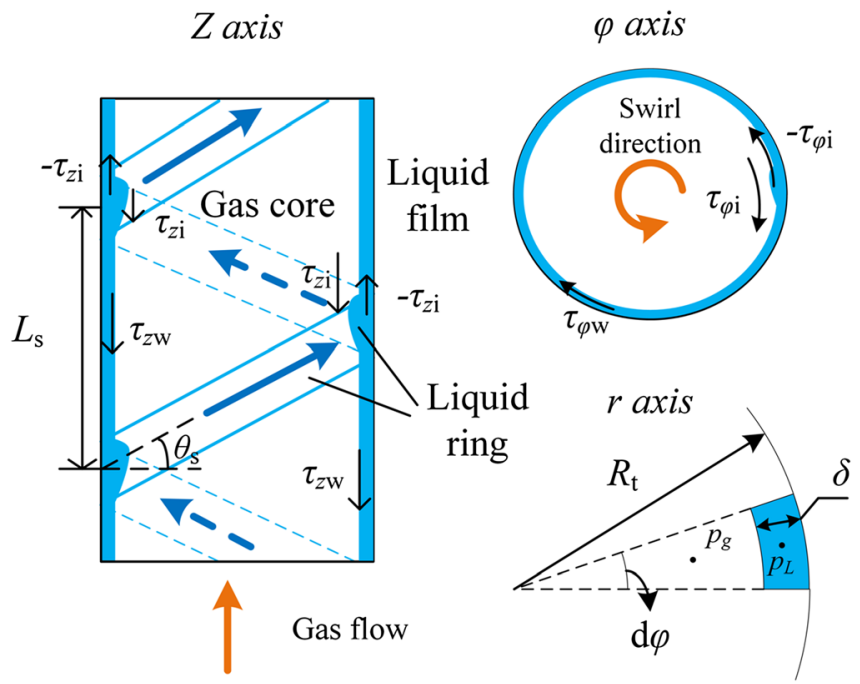

Fig. 1. The schematic diagram of force analysis for gas-liquid flow. two-phase flow study ( $\mathrm{Li}$ et al., 2017), just the same in the vortex flow model. Due to the existence of vortex flow, this model not only take into account the axial mechanical balance, but also the circumferential and radial mechanical balance. For the simplicity of the model, heat transfer between liquid and gas has been ignored. Therefore, the vortex model is for an isothermal flow; the liquid and gas in this model are assumed to be incompressible.

In $r$ direction (radial direction), the radial velocities of gas core and liquid film are both zero. Due to the existence of angular velocity, the centripetal acceleration of liquid makes liquid film pressure higher than the gas core pressure in cross section. The Navier-Stokes (N-S) equation of cylindrical coordinate system under the condition that radial velocity $u_{\mathrm{r}}=0$ is written as:

$$
\frac{u_{\phi}^{2}}{r}=\frac{1}{\rho} \frac{\partial p}{\partial r} .
$$

The angular velocities of gas phase and liquid phase are constant in the same cross section; $p_{\mathrm{g}}$ and $p_{\mathrm{L}}$ are the average pressure of gas core and liquid film respectively. The integration of equation (1) is:

$$
\begin{gathered}
p_{\mathrm{L}}-p_{\mathrm{g}}=\frac{1}{2}\left(\rho_{\mathrm{g}} \omega_{\mathrm{g}}^{2} \int_{0}^{D 2-\delta} r \mathrm{~d} r+\rho_{\mathrm{L}} \omega_{\mathrm{L}}^{2} \int_{D 2-\delta}^{D 2} r \mathrm{~d} r\right) \\
=\frac{D^{2}}{16}\left[\rho_{\mathrm{g}} \omega_{\mathrm{g}}^{2}\left(1-2 \delta_{\mathrm{D}}\right)^{2}+\rho_{\mathrm{L}} \omega_{\mathrm{L}}^{2} 4\left(\delta_{\mathrm{D}}-\delta_{\mathrm{D}}^{2}\right)\right],
\end{gathered}
$$

where $\omega_{\mathrm{g}}$ and $\omega_{\mathrm{L}}$ are the angular velocities of gas core and liquid film respectively, $\delta$ is liquid film thickness $\left(\delta_{\mathrm{D}}=\delta / D\right)$. In the same time, assuming that the liquid film thickness will not change in one vortex unit.

The vortex flow can be seen as a special annular-mist flow with a gas-liquid interface fluctuation in the axial direction, so the momentum equations of the gas core and liquid film are written as (Barnea, 1986; Petalas and Aziz, 1998):

$$
\begin{gathered}
0=-A_{\mathrm{g}} \frac{\mathrm{d} p_{\mathrm{g}}}{\mathrm{d} z}-\rho_{\mathrm{g}} g A_{\mathrm{g}}-\tau_{\mathrm{zi}} S_{f}, \\
0=-A_{\mathrm{L}} \frac{\mathrm{d} p_{\mathrm{L}}}{\mathrm{d} z}-\rho_{\mathrm{L}} g A_{\mathrm{L}}-\tau_{z \mathrm{w}} S_{w}+\tau_{z \mathrm{i}} S_{f} .
\end{gathered}
$$

Considering the angular momentum balance in the circumferential direction, the loss of gas angular momentum is used for overcoming the torque caused by the friction on the gas-liquid interface. Similarly, the variation of liquid momentum in the axial direction is used for overcoming the torque caused by the interfacial friction and the torque of liquid-wall friction. Therefore, the angular momentum balance written as:

$$
\frac{\mathrm{d}}{\mathrm{d} z} \int_{0}^{D 2-\delta} \rho_{\mathrm{g}} u_{\mathrm{zg}} 2 \pi r \mathrm{~d} r \cdot\left(r^{2} \omega_{\mathrm{g}}\right)=-\tau_{\phi \mathrm{i}} S_{f} \frac{\mathrm{D}}{2}\left(1-2 \delta_{\mathrm{D}}\right),
$$




$$
\begin{aligned}
& \frac{\mathrm{d}}{\mathrm{d} z} \int_{D 2-\delta}^{D 2} \rho_{\mathrm{L}} u_{\mathrm{zL}} 2 \pi r \mathrm{~d} r \cdot\left(r^{2} \omega_{\mathrm{L}}\right) \\
& \quad=-\tau_{\phi \mathrm{w}} S_{w} \frac{D}{2}+\tau_{\phi \mathrm{i}} S_{f} \frac{D}{2}\left(1-2 \delta_{\mathrm{D}}\right) .
\end{aligned}
$$

The vortex intensity of gas phase and liquid phase are defined as dimensionless variable $W_{\mathrm{g}}$ and $W_{\mathrm{L}}$ respectively, which are as same as the Liu and Bai (2015) proposed. Furthermore, the variable $\Gamma_{g}$ and $\Gamma_{\mathrm{L}}$ are defined as follow:

$$
\begin{gathered}
W_{\mathrm{g}}=\frac{D\left(1-2 \delta_{\mathrm{D}}\right) \omega_{\mathrm{g}}}{2 u_{\mathrm{zg}}}, \\
W_{\mathrm{L}}=\frac{D \omega_{\mathrm{L}}}{2 u_{\mathrm{zL}}}, \\
\Gamma_{\mathrm{g}}=\frac{\tau_{\phi \mathrm{i}} S_{f}}{A_{\mathrm{g}}}, \\
\Gamma_{\mathrm{L}}=\frac{\tau_{\phi \mathrm{w}} S_{\mathrm{w}}-\tau_{\phi \mathrm{i}} S_{\mathrm{f}}\left(1-2 \delta_{\mathrm{D}}\right)}{A_{\mathrm{L}}} .
\end{gathered}
$$

Combined with equations (7)-(10):

$$
\begin{gathered}
\frac{\mathrm{d} W_{\mathrm{g}}}{\mathrm{d} z}=\frac{-2 \Gamma_{\mathrm{g}}}{\rho_{\mathrm{g}} u_{\mathrm{zg}}^{2}}, \\
\frac{\mathrm{d} W_{\mathrm{L}}}{\mathrm{d} z}=\frac{-\Gamma_{\mathrm{L}}}{\rho_{\mathrm{L}} u_{\mathrm{zL}}^{2}} \frac{1}{\left(1-2 \delta_{\mathrm{D}}+2 \delta_{\mathrm{D}}^{2}\right)} .
\end{gathered}
$$

\subsection{Average liquid film thickness $\delta$}

Combine equations (2)-(4), eliminating $p_{\mathrm{g}}$ and $p_{\mathrm{L}}$ as following:

$$
-\frac{\tau_{\mathrm{zw}} S_{\mathrm{w}}}{A_{\mathrm{L}}}+\tau_{\mathrm{zi}} S_{\mathrm{f}}\left(\frac{1}{A_{\mathrm{g}}}+\frac{1}{A_{\mathrm{L}}}\right)-\left(\rho_{\mathrm{L}}-\rho_{\mathrm{g}}\right) g-W=0 .
$$

Equation (12) is used to calculate the average liquid film thickness $\delta$. $W$ reflects the effect of centrifugal force on $\delta$ caused by vortex flow. Experiment shows that the vortex flow will gradually disappear along the pipeline which expresses as $\mathrm{d} W / \mathrm{d} z<0$. If $W=0$, equation (10) will be same as the common annular-mist model (Ansari et al., 1990; Taitel and Dukler, 1976). $W$ is calculated by equation (14):

$$
W=-\Gamma_{\mathrm{g}} W_{\mathrm{g}}-\frac{2\left(\delta_{\mathrm{D}}-\delta_{\mathrm{D}}^{2}\right)}{1-2 \delta_{\mathrm{D}}+2 \delta_{\mathrm{D}}^{2}} \Gamma_{\mathrm{L}} W_{\mathrm{L}} .
$$

In this equation, $W<0$ means the liquid film thickness is smaller than that of common annular-mist model. Therefore, vortex flow will decrease the loss of gravitational pressure, which is one of the mechanisms of the vortex tools.

\subsection{The interfacial relations}

The axial shear stress and circumferential shear stress are written as follows:

$$
\tau_{\mathrm{zw}}=0.5 f_{\mathrm{zw}} \rho_{\mathrm{L}} u_{\mathrm{zL}}^{2}
$$

$$
\begin{gathered}
\tau_{\phi \mathrm{w}}=0.5 f_{\phi \mathrm{w}} \rho_{\mathrm{L}}\left(W_{\mathrm{L}} u_{\mathrm{zL}}\right)^{2}, \\
\tau_{\mathrm{zi}}=\tau_{\mathrm{gf}}\left[1+\frac{2(\eta-1) h_{\mathrm{aD}}}{\pi}\right], \\
\tau_{\phi \mathrm{i}}=0.5 f_{\phi i} \rho_{\mathrm{g}}\left(W_{\mathrm{g}} u_{\mathrm{zg}}\right)^{2} .
\end{gathered}
$$

where $\tau_{\mathrm{zw}}, \tau_{\varphi \mathrm{w}}, \tau_{\mathrm{zi}}$ and $\tau_{\varphi \mathrm{i}}$ are the axial liquid-wall shear stress, the circumferential liquid-wall shear stress, axial liquid-gas interfacial shear stress and circumferential liquid-gas interfacial shear stress, respectively. $\tau_{\mathrm{gf}}$ in equation (17) is the liquid-gas interfacial shear stress in the common annular flow, which can be calculated by equation (29). Because of the existence of vortex tool, liquid will converge into the vortex channel and form a liquid ring that flows upward along the pipeline. The protruding liquid ring occupies some cross section of the gas core. Therefore, the liquid-gas interfacial shear stress will produce a drag force on the liquid ring. In order to simplify the model, it is assumed that the shear stress is equal to the drag force. The result shows that $\eta>1$ and $\tau_{\mathrm{zi}} \geq \tau_{\mathrm{gf}}$, which means under the effect of vortex tool, the drag force on liquid increases, and the thickness of liquid film decreases calculated by equation (13), which will lead to the decrease of gravitational pressure drop.

The relations of friction factor $f$ are shown as follows:

$$
f_{\mathrm{zw}}=0.046 \mathrm{Re}_{\mathrm{L}}^{-0.2},
$$

$$
\begin{gathered}
f_{\mathrm{zi}}=0.24 f_{\mathrm{g}} \operatorname{Re}_{\mathrm{L}}^{0.305}\left[\frac{\sigma}{D \rho_{\mathrm{g}} u_{\mathrm{g}}^{2}\left(1-2 \delta_{\mathrm{D}}\right)}\right]^{0.085}, \\
f_{\phi \mathrm{w}}=0.0175 f_{\mathrm{w}} W_{\mathrm{L}}^{-0.294}, \\
f_{\phi \mathrm{i}}=0.0175 f_{\mathrm{g}} W_{\mathrm{g}}^{-0.294},
\end{gathered}
$$

where axial liquid-gas interfacial friction factor $f_{\mathrm{zi}}$ was proposed by Wei et al. (2014), $u_{\mathrm{g}}$ in equation (17) is defined as $u_{\mathrm{g}}=u_{\mathrm{zg}}\left(1+W_{\mathrm{g}}^{2}\right)^{0.5}$. 


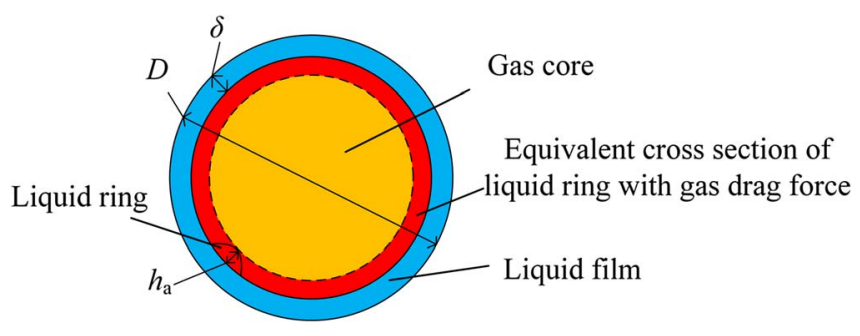

Fig. 2. The equivalent force cross section of the liquid ring dragged by gas in the vortex flow.

\subsection{Calculation of shear stresses}

When gas core flows through the protruding liquid ring, there is a drag force on the liquid ring, which is calculated as:

$$
F_{\text {drag }}=0.5 C_{\mathrm{D}} \rho_{\mathrm{g}} u_{\mathrm{r}}^{2} A_{\text {drag }} .
$$

In the vortex flow, the total cross-section area of protruding liquid ring is shown in Figure 2 with red part, assuming the drag force equals to the shear stress on liquid ring:

$$
\begin{gathered}
\tau_{\mathrm{r}}=\frac{F_{\text {drag }}}{A_{\text {shear }}}, \\
A_{\text {drag }}=\pi h_{\mathrm{a}}\left[D\left(1-2 \delta_{\mathrm{D}}\right)-h_{\mathrm{a}}\right], \\
A_{\text {shear }}=2 h_{\mathrm{a}} \pi D\left(1-2 \delta_{\mathrm{D}}\right) \sqrt{1+\frac{1}{W_{\mathrm{L}}^{2}}} .
\end{gathered}
$$

In order to compare with the common two-phase annular flow, equation (24) can be rewritten as:

$$
\begin{gathered}
\tau_{\mathrm{r}}=\eta \tau_{\mathrm{gf}} \\
\eta=\frac{C_{\mathrm{D}}\left(1-h_{\mathrm{aD}}\right)}{2 f_{\mathrm{zi}}} \sqrt{\frac{W_{\mathrm{L}}^{2}}{1+W_{\mathrm{L}}^{2}}} .
\end{gathered}
$$

In equation $(27), \tau_{\mathrm{gf}}$ indicates the shear stress between gas core and liquid film without liquid ring, which is calculated as:

$$
\tau=0.5 f_{\mathrm{zi}} \rho_{\mathrm{g}}\left(u_{\mathrm{zg}}-u_{\mathrm{zL}}\right)^{2} .
$$

In equation $(28), C_{\mathrm{D}}$ is drag coefficient proposed by Morsi and Alexander (1972):

$$
C_{\mathrm{D}}=a+b \mathrm{Re}^{-1}+c \mathrm{Re}^{-2},
$$

where Reynold number is defined as $\operatorname{Re}=\rho_{\mathrm{g}} u_{\mathrm{zg}} h_{\mathrm{a}} / \mu_{\mathrm{g}}$.

The forces applied to the liquid phase include the drag force on the liquid ring and shear stress on the liquid film, so the resultant force is written as:

$$
\begin{aligned}
\tau_{\mathrm{zi}} & =\left(1-\frac{2 h_{\mathrm{a}}}{\pi D\left(1-2 \delta_{\mathrm{D}}\right)}\right) \tau_{\mathrm{gf}}+\frac{2 h_{\mathrm{a}}}{\pi D\left(1-2 \delta_{\mathrm{D}}\right)} \tau_{\mathrm{r}} \\
& =\tau_{\mathrm{gf}}\left[1+\frac{2(\eta-1) h_{\mathrm{aD}}}{\pi}\right]
\end{aligned}
$$

where $h_{\mathrm{a}}$ is the thickness of liquid ring, defining $h_{\mathrm{aD}}$ as: $h_{\mathrm{aD}}=h_{\mathrm{a}} /\left(D\left(1-2 \delta_{\mathrm{D}}\right)\right)$. Ryu and Park (2011) proposed a correlation to calculate the interface fluctuation amplitude in two-phase annular flow, which is introduced into present work, empirical parameter $h_{\mathrm{aD}}$ is calculated as:

$$
h_{\mathrm{aD}}=a C_{\mathrm{w}} \operatorname{Re}_{\mathrm{sL}}^{\mathrm{b}}
$$

$a$ and $b$ are determined to 18 and -0.8 respectively; $C_{\mathrm{w}}$ is a function of gas and liquid physical property which is defined as follow:

$$
\begin{gathered}
\left\{\begin{array}{c}
\frac{1}{3 C_{\mathrm{w}}}=11.78 \mathrm{~N}_{\mu}^{0.8}, \quad N_{\mu} \leq \frac{1}{15} \\
\frac{1}{3 C_{\mathrm{w}}}=1.35, \quad N_{\mu}>\frac{1}{15}
\end{array}\right. \\
N_{\mu}=\frac{\mu_{\mathrm{L}}}{\sqrt{\rho_{\mathrm{L}} \sigma \sqrt{\frac{\sigma}{g\left(\rho_{\mathrm{L}}-\rho_{\mathrm{g}}\right)}}}} .
\end{gathered}
$$

\subsection{Solution for the model}

According to the mass balance law, the mass flow rate of gas and the mass flow rate of liquid are constant. Thus, the axial average velocity of gas and liquid are calculated as:

$$
\begin{aligned}
& u_{\mathrm{zg}}=\frac{A_{\mathrm{t}}}{A_{\mathrm{g}}} u_{\mathrm{sg}}, \\
& u_{\mathrm{zL}}=\frac{A_{\mathrm{t}}}{A_{\mathrm{L}}} u_{\mathrm{sL}},
\end{aligned}
$$

where $A$ is cross-section area; the subscripts $t, r$ and $f$ denote the test pipeline, liquid ring and liquid film, respectively.

$$
\begin{gathered}
A_{\mathrm{t}}=\frac{1}{4} \pi D^{2}, \\
A_{\mathrm{L}}=A_{\mathrm{f}}+A_{\mathrm{r}}=\pi D^{2}\left(\delta_{\mathrm{D}}-\delta_{\mathrm{D}}^{2}\right)+\pi h_{\mathrm{a}}^{2}, \\
A_{\mathrm{g}}=A_{\mathrm{t}}-A_{\mathrm{L}}=\frac{1}{4} \pi D^{2}\left(1-2 \delta_{\mathrm{D}}\right)^{2}-\pi h_{\mathrm{a}}^{2}, \\
S_{\mathrm{fi}}=\pi D\left(1-2 \delta_{\mathrm{D}}\right), \\
S_{\mathrm{f}}=\pi D,
\end{gathered}
$$

where $S_{\text {fi }}$ is the wetted perimeter between liquid film and pipe wall, $S_{\mathrm{f}}$ is the interfacial perimeter. The liquid holdup of cross section is shown as: 


$$
H_{\mathrm{L}}=4\left(\delta_{\mathrm{D}}-\delta_{\mathrm{D}}^{2}\right)+h_{\mathrm{aD}}^{2}\left(1-2 \delta_{\mathrm{D}}\right)^{2} .
$$

When calculating the pressure profile along the pipeline, it is needed to segment the pipe into units, and choose the appropriate method to calculate the pressure gradient of each unit (Fu et al., 2015; Zhang and Li, 2011), then the outlet pressure can be obtained. The pressure calculation procedure is shown in Figure 3.

\section{The two-phase flow experiments with vortex tools}

\subsection{The experiments arrangement}

Air and water are regarded as gas phase flow medium and liquid phase flow medium, respectively. The experimental flow loop is shown in Figure 4. Water is injected through the screw pump, and the flow rate ranges from $0.1 \mathrm{~m}^{3} / \mathrm{h}$ to $6.3 \mathrm{~m}^{3} / \mathrm{h}$. Air is supplied through the compressor whose maximum rate is $5 \mathrm{~m}^{3} / \mathrm{min}$, and maximum outlet pressure is $1.2 \mathrm{MPa}$. A vertical transparent test section is set to observe the vortex flow, and different vortex tools can be changed at the bottom of the section. The length of test section is $7.5 \mathrm{~m}$, which is constituted of stainless-steel pipe and transparent pipe. There are three vortex tools with different helical angles as shown in Figure 5 (angle between liquid film ring and horizontal plane), which are $30^{\circ}, 45^{\circ}$ and $55^{\circ}$ respectively, to simulate different vortex flow conditions.

\subsection{Flow parameter measurement}

Three Rosemount pressure gauges are installed below and above the vortex tool, and at the outlet of test pipeline with the range of $0-500 \mathrm{kPa}$, they are used to measure pressure drop. Several flowmeters are also installed on the pipeline, including a turbine flowmeter for the water volume flow rate with a range of $0.1-1.5 \mathrm{~m}^{3} / \mathrm{h}$; a gas volume flowmeter with a range of 15-3000 L/min. Helical liquid film can be observed on the transparent section after the vortex tool. Based on the helical angle of liquid film ring, the vortex intensity can be obtained. According to the experiments, it is found that liquid velocity drops because of the friction between the liquid film and pipe wall, which can be reflected by the increasement of helical angle.

\subsection{Experiments procedure}

Firstly, air is injected from compressor into the gas tank, and input the gas to the pipe with a fixed flow rate. When the gas flow reached the stability in the whole pipeline, injected water into the mixer under a low rate from the water tank, gradually increased the water flow rate until it formed a liquid ring. The gas-water flow must reach a steady state for several minutes, and recorded the pressure data, gas velocity and liquid velocity. Repeated the above steps with different gas flow rate, liquid flow rate and different vortex tools.

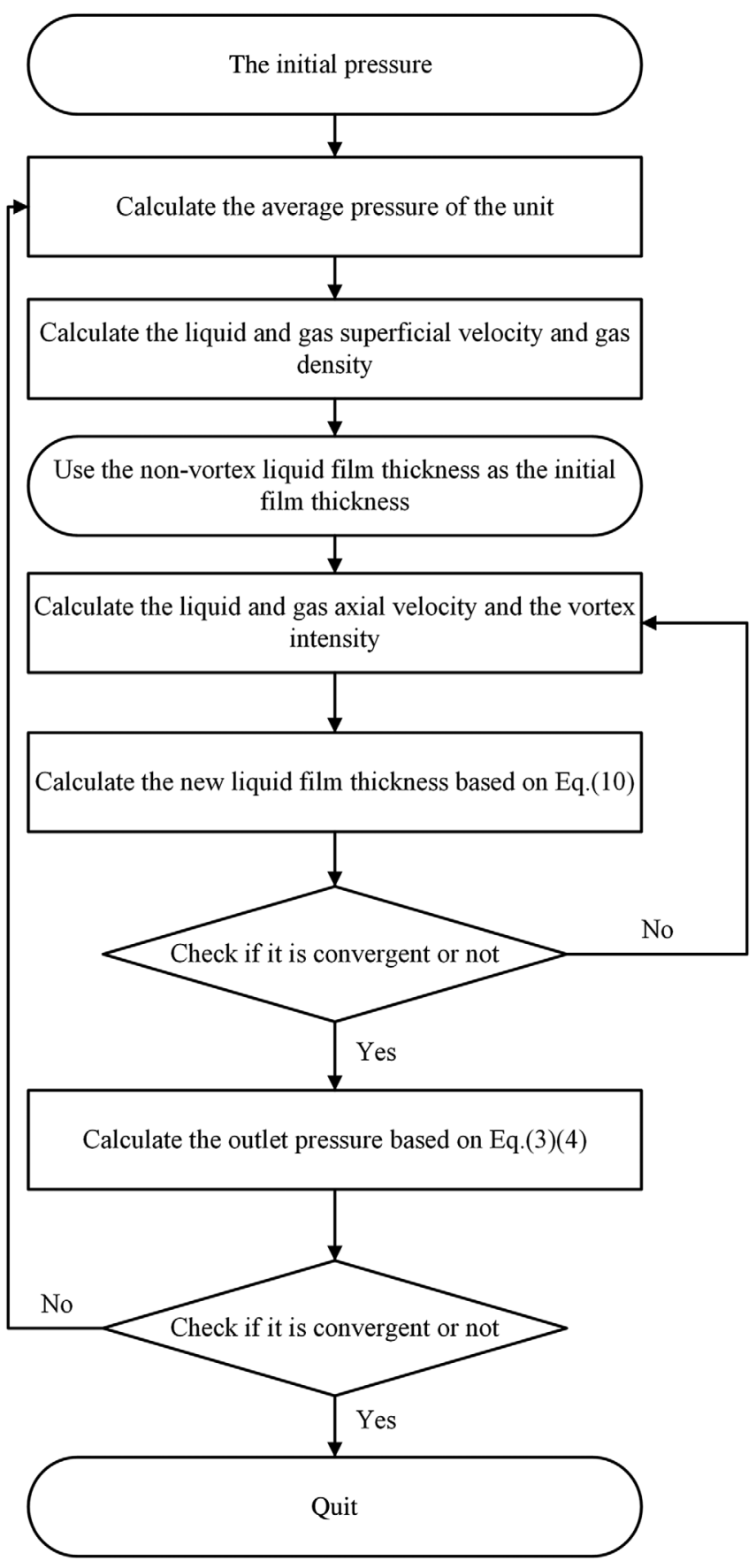

Fig. 3. The flow-process diagram of pressure calculation in a unit.

\section{Validate results and discussion}

\subsection{Results comparison between model and previous experiments}

Ali et al. (2005) studied the critical gas velocity with or without the vortex tool, and he obtained the two-phase pressure drop under critical condition. The present work selects the annular flow experimental results, as shown in 


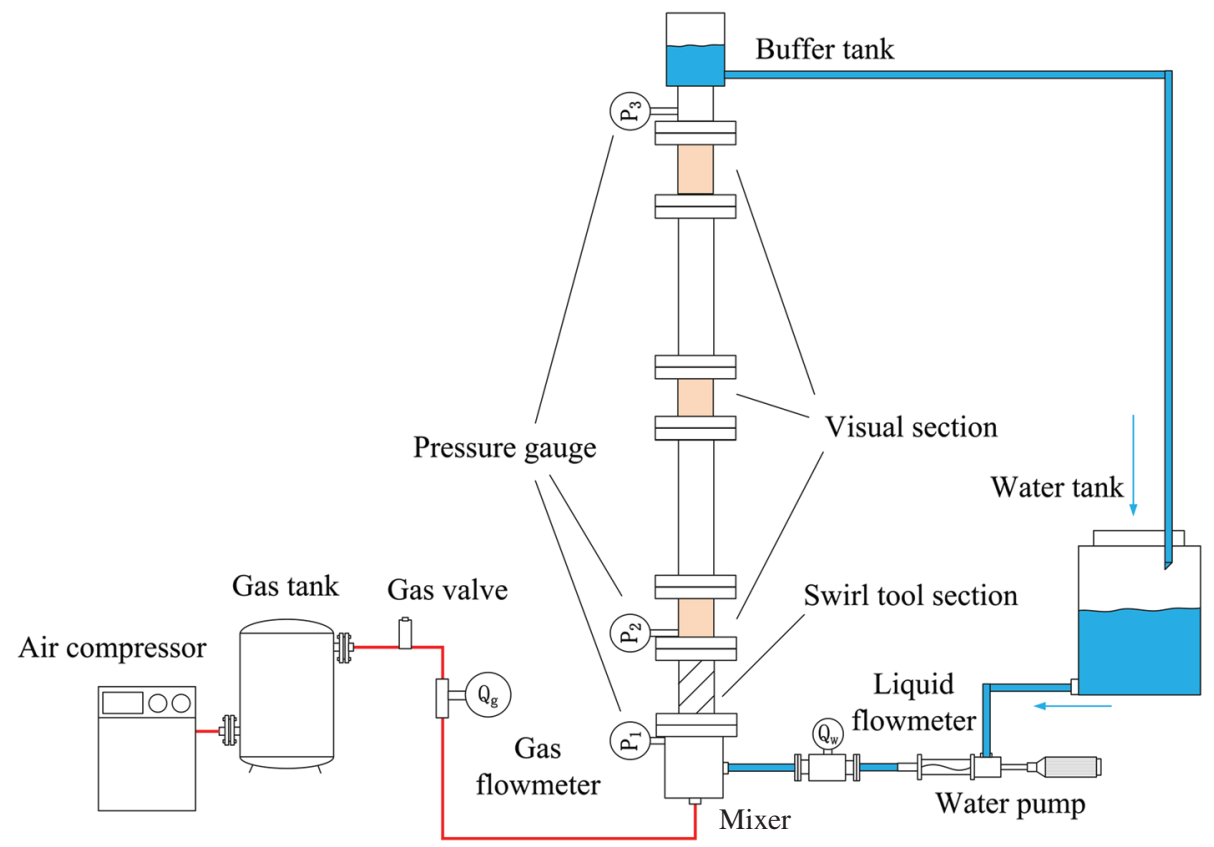

Fig. 4. The flow loop of gas-liquid vertical vortex flow experiments.

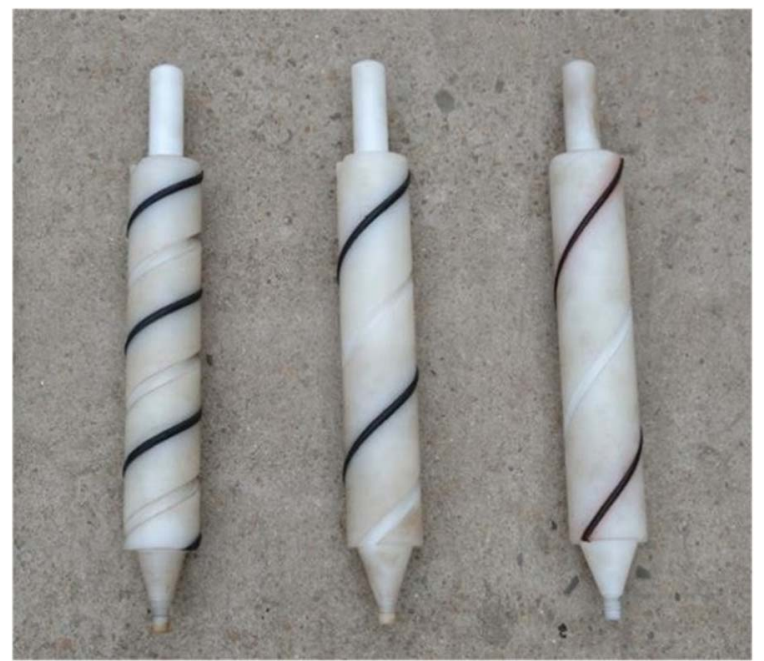

Fig. 5. The vortex tools used in the experiments.

Figure 6 (scattering points), it is found that the pressure drops with vortex tool (solid points) is smaller than the pressure drops without vortex tool (hollow points) under same gas velocity. When the outlet pressures are $107.27 \mathrm{kPa}, 239.22 \mathrm{kPa}$ and $308.17 \mathrm{kPa}$, the average decrease of pressure drop are $17.0 \%, 8.5 \%$ and $5.0 \%$, respectively. Comparing the vortex flow model with three of the most common models used in the multiphase flow calculation in oil field, which are Beggs-Brill (B \& B) model, Hagedorn-Brown (H \& B) model and Ansari model, as shown in Figures 6-8. Because Ali et al. (2005) did not give the vortex intensity in their study, the vortex intensity used to calculate the vortex flow pressure drop in present research is assumed as 0.88 . In Figure 6, when the inlet

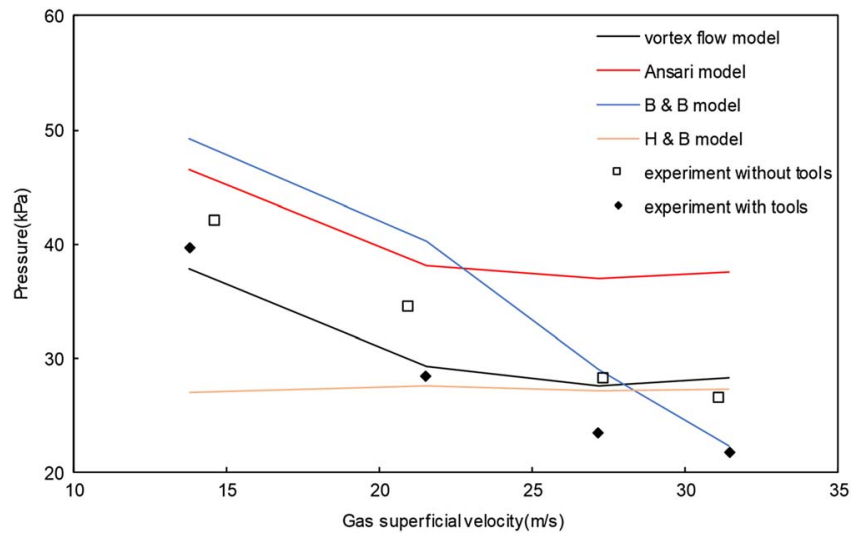

Fig. 6. Pressure drop of gas-liquid two-phase vortex flow under the critical liquid unloading condition (inlet pressure $170.27 \mathrm{kPa}$ ).

pressure is $170.27 \mathrm{kPa}$, it is found that $\mathrm{B} \& \mathrm{~B}$ model and $\mathrm{H} \& \mathrm{~B}$ model have large differences with the experimental results; the vortex flow model and the Ansari model have the same trend as the experiments, but Ansari model fits better to the non-vortex experimental results. When the gas superficial velocity is below $23 \mathrm{~m} / \mathrm{s}$, vortex flow model fits well to vortex experimental results; when the gas superficial velocity reaches $23 \mathrm{~m} / \mathrm{s}$, the vortex flow model results become larger than vortex experimental results. In Figure 7, when the inlet pressure is $239.22 \mathrm{kPa}$, it can be obtained that all models have rather good trends, as gas superficial velocity reaches $25 \mathrm{~m} / \mathrm{s}$, other models have large differences, but the vortex flow model performs well all the time. In Figure 8, when the inlet pressure is $308.17 \mathrm{kPa}, \mathrm{B} \& \mathrm{~B}$ model and $\mathrm{H} \& \mathrm{~B}$ model are significantly lower than the experimental results, and Ansari model is higher than the 


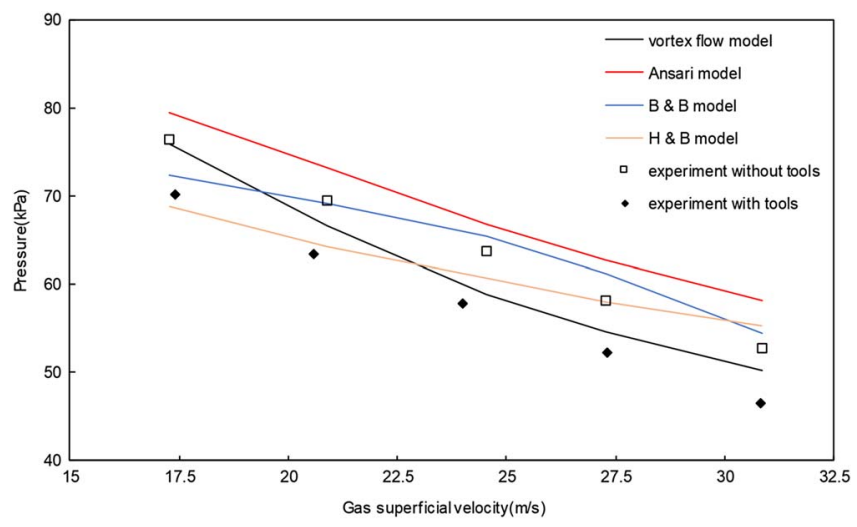

Fig. 7. Pressure drop of gas-liquid two-phase vortex flow under the critical liquid unloading condition (inlet pressure $239.22 \mathrm{kPa})$

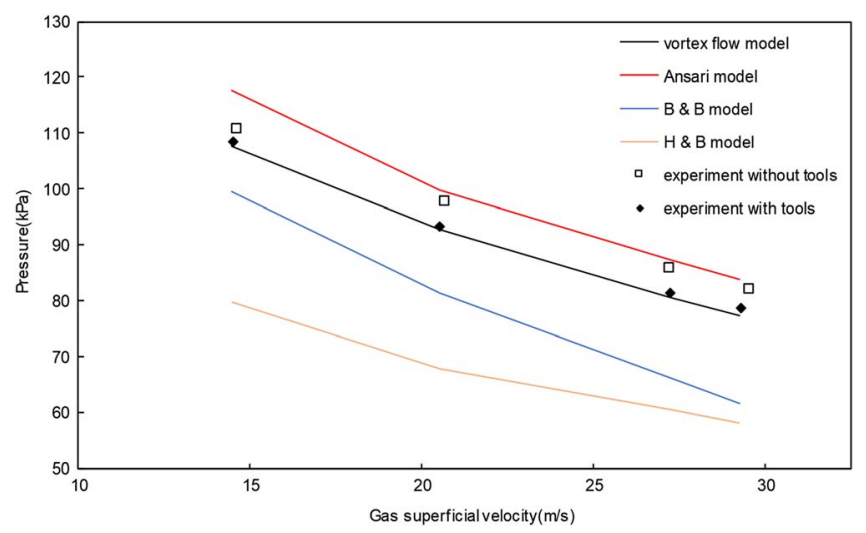

Fig. 8. Pressure drop of gas-liquid two-phase vortex flow under the critical liquid unloading condition (inlet pressure $308.17 \mathrm{kPa})$.

vortex experimental results, only vortex flow model fits very well. Generally, Ansari model and vortex flow model have better accuracies than other models; but under the conditions of three different outlet pressure, pressure drops of vortex flow model are $22.87 \%, 10.42 \%$ and $7.83 \%$ lower than those of Ansari model, which indicates that the vortex flow model can better reflect the effect of vortex tool.

The maximum relative differences between vortex flow model and experiments are $30.06 \%, 8.10 \%$ and $1.76 \%$, respectively; the average relative differences between vortex flow model and experiments are $11.54 \%, 5.41 \%$ and $1.08 \%$, respectively. In addition, it can be found that this model performs better when the inlet pressure is higher.

\subsection{Results comparison between vortex flow model and pressure drop experiments}

In the preceding section, Ali et al. (2005) only gave the common experimental results, they didn't come up with the parameters related to vortex flow, which is of great importance for liquid unloading process. Their study mainly focused on the promotion of liquid unloading capacity,
Table 1. Experimental conditions of gas-liquid twophase swirl flow.

\begin{tabular}{lcc}
\hline Experimental conditions & Ali & Present work \\
\hline Pipe diameter (mm) & 50.8 & 50 \\
Pipe length (m) & 38.1 & 7.5 \\
Liquid vortex flow & $/$ & $1.73,1,0.70$ \\
intensity & Air and & Air and \\
Media & water & water \\
& $170 \sim 310$ & $150 \sim 200$ \\
Inlet pressure (kPa) & $13.5 \sim 31.5$ & $12.0 \sim 18.0$ \\
Gas superficial velocity & & \\
(m/s) & $0.05 \sim 0.60$ & $0.05 \sim 0.20$ \\
Liquid superficial & & \\
velocity (m/s) & Critical unloading & Unloading \\
Flow condition & state & state \\
\hline
\end{tabular}

but it lacked of experiments of condition that over critical unloading velocity. For further validating the vortex flow model, there were some more advanced experiments conducted in the Key Laboratory of Petroleum Engineering of China University. The experimental conditions are shown in Table 1.

The helical angle of the liquid ring $\theta_{\mathrm{s}}$ can be measured on the transparent test sections, based on which the liquid vortex flow intensity is calculated as:

$$
\frac{1}{\tan \theta_{\mathrm{s}}}=\frac{u_{\phi \mathrm{L}}}{u_{\mathrm{zL}}}=\frac{D \omega_{\mathrm{L}}}{2 u_{\mathrm{zL}}}=W_{\mathrm{L}} .
$$

Gas-water mixture flows through the vortex tool channel, and the vortex flow intensity is determined by the helical angle when the mixture flowed out of tools. In present experiments, the helical angles of vortex tool are $30^{\circ}, 45^{\circ}$ and $55^{\circ}$, the corresponding vortex flow intensity are 1.73 , 1 and 0.70 . The pressure drops against gas superficial velocities under three kinds of liquid superficial velocities with vortex tool $\# 1, \# 2$ and $\# 3$ are plotted in Figures 9-11 respectively. In these graphics, scattering points are experimental data. As the gas superficial velocity increases, the cross-section liquid holdup and pressure drop decrease; but when the gas superficial velocity becomes higher, it will lead to the higher friction that cause the pressure drop increases. When liquid superficial velocity is $0.05 \mathrm{~m} / \mathrm{s}$, it is shown in Figure 9 that the pressure drop decreases from $18.21 \mathrm{kPa}$ to $7.51 \mathrm{kPa}$, then rises to $10.51 \mathrm{kPa}$ with the increase of gas superficial velocity. Under the condition of high liquid superficial velocity, the effect of decreasing liquid holdup is more obvious, so the pressure drop decreases continuously, but under low liquid velocity condition, the pressure drop inversion is not obvious. It can be found in Figures 10 and 11 that the pressure drops trend of vortex tool $\# 2$ and $\# 3$ are similar to tool $\# 1$. In Figures 9-11, the data analysis of tool \#1 shows that the maximum and minimum relative differences between vortex model and experiments data are $31.7 \%$ and $0.1 \%$, respectively; and the average difference between vortex model and 


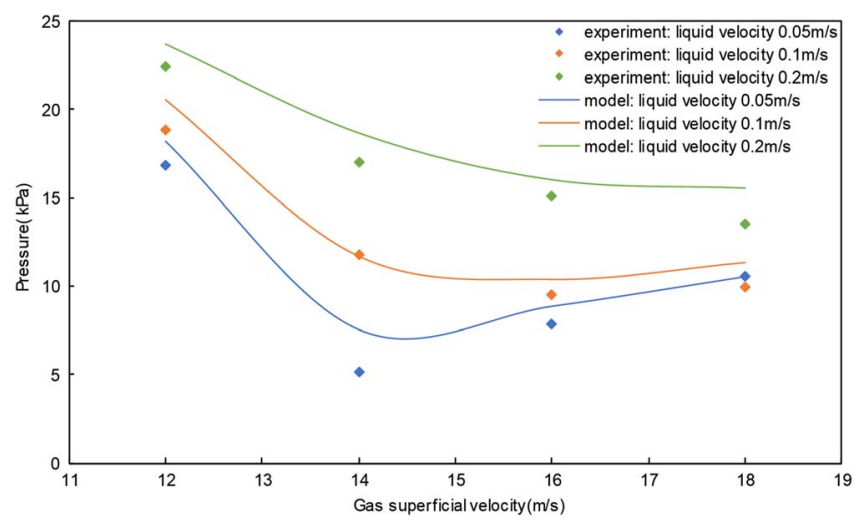

Fig. 9. Experimental and theoretical results of pressure drop and velocity of gas-liquid vortex flow (vortex tool $\# 1$ ).

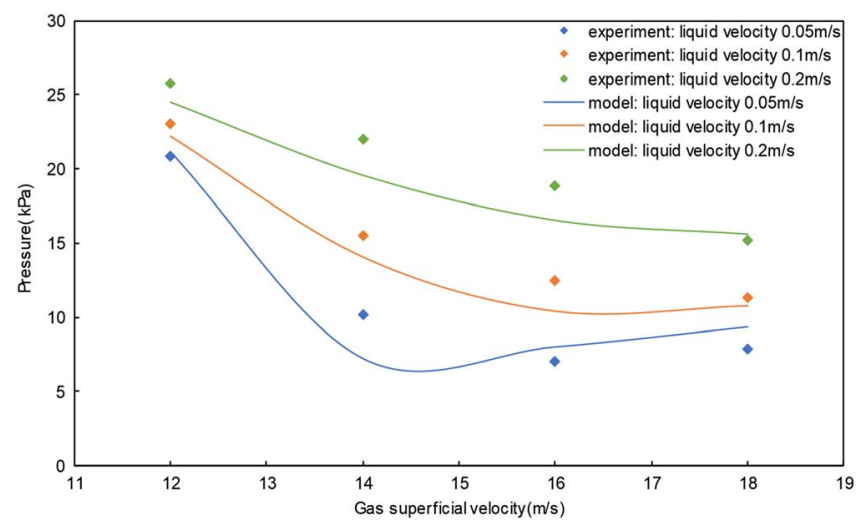

Fig. 10. Experimental and theoretical results of pressure drop and velocity of gas-liquid vortex flow (vortex tool \#2).

experiments data is $9.6 \%$. The analysis of tool $\# 2$ shows that the maximum and minimum relative differences between vortex model and experiments data are 29.3\% and $2.5 \%$, respectively; and the average difference between vortex model and experiments data is $10.7 \%$. The analysis of tool \#3 shows that the maximum and minimum relative differences between vortex model and experiments data are $15.6 \%$ and $0.2 \%$, respectively; and the average difference between vortex model and experiments data is $7.5 \%$. Generally, this model is well fitted to experimental results.

Figure 12 shows the pressure drops against gas superficial velocity with different vortex tools when the liquid superficial velocity is $0.05 \mathrm{~m} / \mathrm{s}$. It can be found that the helical angle of vortex tool has contradictory influences on the two-phase flow under different gas superficial velocity conditions. When gas superficial velocity is low $(12 \mathrm{~m} / \mathrm{s}-14 \mathrm{~m} / \mathrm{s})$, the pressure drop increases as the helical angle becomes bigger; when gas superficial velocity is high $(16 \mathrm{~m} / \mathrm{s}-18 \mathrm{~m} / \mathrm{s})$, the pressure drop decreases as the helical angle becomes bigger. For example, the vortex tool \#1 has the smallest helical angle, according to equation (43), which leads to the biggest vortex flow intensity; and based on equation (31), it has the biggest interfacial force, so liquid holdup will decrease at low gas superficial velocity, while

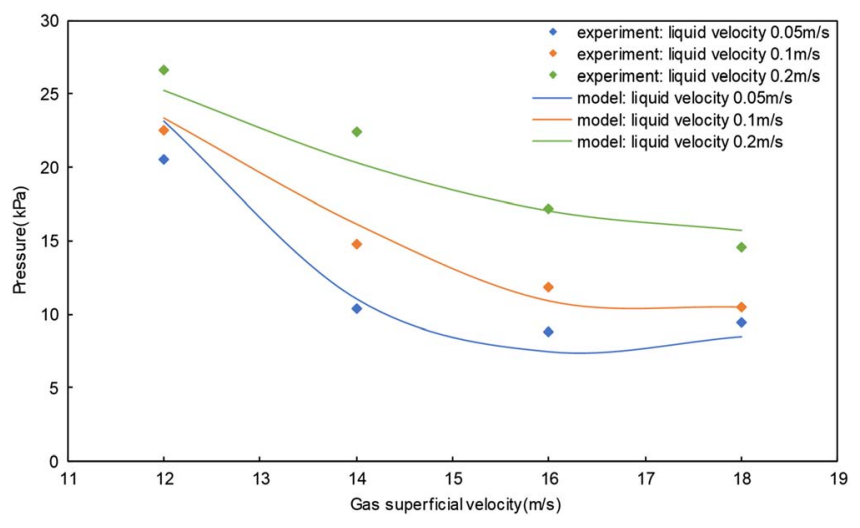

Fig. 11. Experimental and theoretical results of pressure drop and velocity of gas-liquid vortex flow (vortex tool \#3).

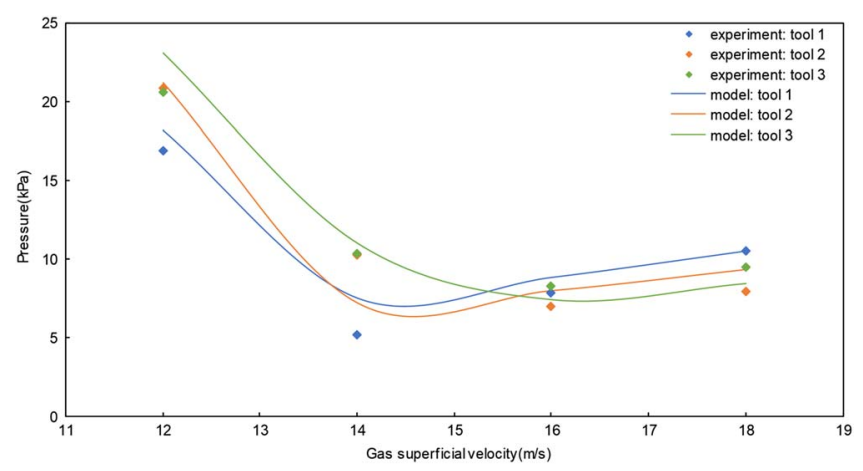

Fig. 12. The results of different vortex tools of pressure drop and velocity (at the liquid velocity of $0.05 \mathrm{~m} / \mathrm{s}$ ).

increase friction at high gas superficial velocity. Figure 12 also shows that the minimum pressure drop is $15 \%-35 \%$ lower than the maximum pressure drop with different vortex tools, therefore when using vortex tools in a gas well, actual flow conditions should be considered.

\subsection{Results comparison between vortex flow model and decay experiments of vortex flow intensity}

The angular velocities of gas and liquid will gradually decrease caused by interfacial frictions. The average vortex flow intensity gradient $\mathrm{d} W_{\mathrm{L}} / \mathrm{d} z$ in the test section is calculated based on the helical angle and the vortex flow intensities at the inlet and outlet of vortex tool. Figures 13-15 show the theoretical and experimental liquid vortex flow intensities under different conditions. Both experimental and calculated results show that vortex flow intensity decay rate decreases along the pipe as liquid velocity increases. There also is a rising trend of $-\mathrm{d} W_{\mathrm{L}} / \mathrm{d} z$ as gas velocity increases in Figures 13-15, which due to the liquid film thickness decreased caused by rising gas velocity. The rise of vortex flow intensity decay rate is based on equation (13). In Figures 13-15, the differences between experimental data and calculation results are large under low liquid superficial velocity condition; then, the differences become smaller under high liquid superficial velocity, mainly because the vortex flow has fully developed. 


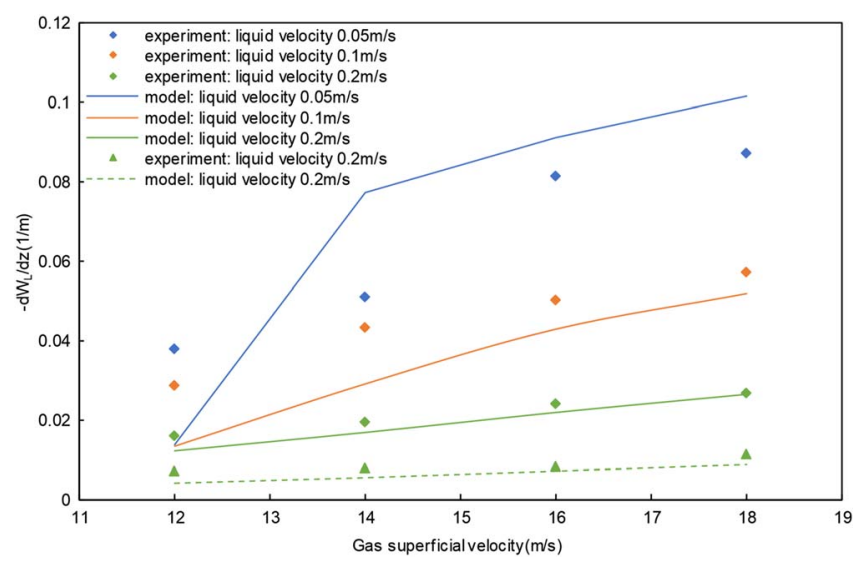

Fig. 13. The experimental and theoretical results of the liquid vortex flow intensity (The series of $\bullet$ and solid lines represent the results of the tool $\# 1$. The series of $\boldsymbol{\Delta}$ and dotted lines represent the results of tool $\# 3$ ).

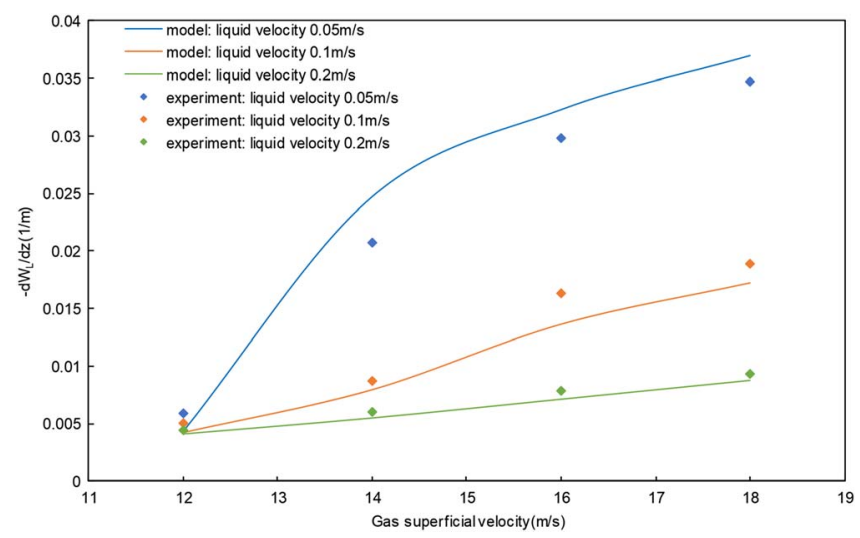

Fig. 14. The experimental and theoretical results of the liquid vortex flow intensity of tool $\# 2$.

It can be found from Figures 13-15 that vortex intensity gradients have similar trend as gas superficial velocity increases; the $-\mathrm{d} W_{\mathrm{L}} / \mathrm{d} z$ drops rather obviously as the helical angle becomes higher with different vortex tools. In Figures 13-15, the maximum and minimum relative differences between vortex model and experiments data of tool $\# 1$ are $63.3 \%$ and $2.1 \%$, respectively; and the average difference is $25.3 \%$. The maximum and minimum relative differences between vortex model and experiments data of tool $\# 2$ are $37.8 \%$ and $6.0 \%$, respectively; and the average difference is $13.9 \%$. The maximum and minimum relative differences between vortex model and experiments data of tool $\# 3$ are $9.0 \%$ and $2.1 \%$, respectively; and the average difference is $4.1 \%$, the calculation results of vortex model is acceptable. According to the green solid line and dotted line in Figure 13, which represent $-\mathrm{d} W_{\mathrm{L}} / \mathrm{d} z$ of tool $\# 1$ and tool \#3 respectively. It can be found that the lower initial velocity causes the smaller friction between liquid and pipe wall, which will lead to the smaller vortex flow intensity gradient. Based on this conclusion, it can be inferred that the vortex flow intensity will decrease upward along the pipe and the decrease rate will gradually become smaller.

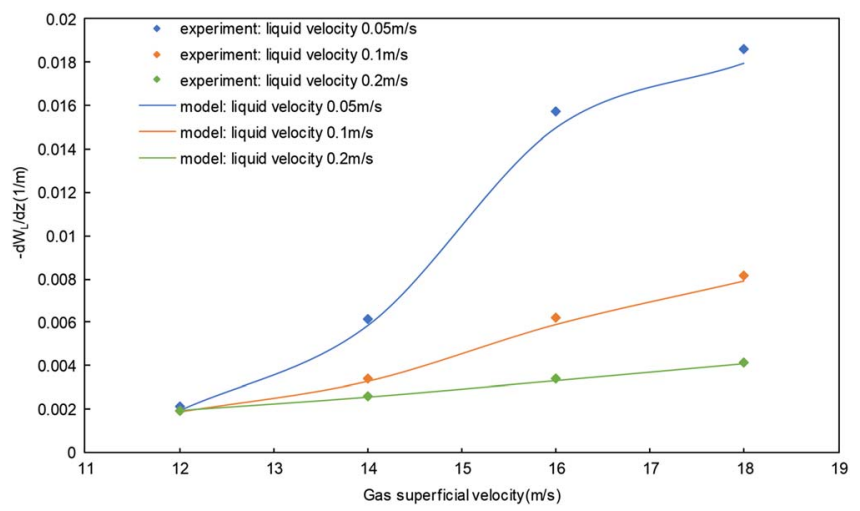

Fig. 15. The experimental and theoretical results of the liquid vortex flow intensity of tool \#3.

The vortex flow working distance can be calculated by the vortex flow intensity. For example, when the liquid and gas superficial velocities are $0.05 \mathrm{~m} / \mathrm{s}$ and $16 \mathrm{~m} / \mathrm{s}$, the vortex flow intensity gradient is $0.10 \mathrm{~m}^{-1}$ and the vortex flow intensity of tool $\# 1$ is 1.73 . The theoretical working distance is $17.3 \mathrm{~m}(1.73 / 0.10)$, but the actual working distance is a little larger than $17.3 \mathrm{~m}$. It can be found that the vortex intensity gradient is related to liquid velocity, the relation is that the vortex intensity gradient becomes lower as liquid velocity rises, which leads to the longer working distance. Taking tool $\# 1$ as an example, when liquid velocity is $0.1 \mathrm{~m} / \mathrm{s}$ and the vortex intensity gradient is $0.04 \mathrm{~m}^{-1}$, the working distance is $40 \mathrm{~m}$; when liquid velocity is $0.2 \mathrm{~m} / \mathrm{s}$ and the vortex intensity gradient is $0.02 \mathrm{~m}^{-1}$, the working distance is $86.5 \mathrm{~m}$. However liquid velocity can't increase limitlessly. As the liquid velocity increases, the pressure drop will become higher and the stability of gasliquid interface will decrease, which leads to collapse of liquid ring and causes liquid loading. When the vortex flow intensity is near 0 , the effect for lowing pressure drop is inconspicuous. Therefore, the actual gas production situations should be taken into account when design the interval of vortex tools.

\section{Conclusion}

Under the vortex flow condition, there is a pressure difference between gas core and liquid film due to the centrifugal force in two-phase annular flow caused by circumferential flow. A drag force is added on the liquid phase due to the structural characteristics of liquid ring. Both situations will lead to the two-phase pressure drop decreasing under the vortex flow.

The present study proposes the gas and liquid momentum equations in axial, circumferential and radial directions, which can calculate thickness of liquid film, vortex flow intensity and pressure drop. Comparing the previous experiments and models with vortex flow model, it shows that the vortex flow model performs better than other models, and its average relative differences are $11.54 \%$, $5.41 \%$ and $1.08 \%$ when inlet pressure are $170.27 \mathrm{kPa}$, $239.22 \mathrm{kPa}$ and $308.17 \mathrm{kPa}$, respectively. The analyses 
indicate that the vortex flow model is well fitted to experimental results.

Vortex tools with bigger helical angle will lead to higher vortex flow intensity. As the gas superficial velocity increases, on one side it will lead to the decrease of liquid holdup and pressure drop; on the other side it will lead to the high friction which causes the increasing of pressure drop. Therefore, under high gas velocity condition, higher vortex flow intensity tends to the increase of flow friction; under low gas velocity condition, the trend is inverse.

The vortex flow theoretical working distance can be calculated by the vortex intensity gradient and initial vortex flow intensity. Larger liquid superficial velocity will lead to the longer working distance, but liquid velocity can't increase limitlessly, it might lead to collapse of liquid ring and cause liquid loading.

Acknowledgments. The authors express gratitude for the support of the National Natural Science Foundation of China (Grant No. 51574256) and the National Major Science and Technology Subproject "Critical Technology for Drilling and Completion of Complex Structural Wells and Cluster Wells" (2017ZX05009-003). This work is financially supported by them.

\section{References}

Alekseenko S.V., Kuibin P.A., Okulov V.L., Shtork S.I. (1999) Helical vortices in swirl flow, J. Fluid Mech. 382, 195-243.

Ali A.J., Scott S.L., Fehn B. (2005) Investigation of new tool to unload liquids from stripper-gas wells, SPE Prod. Facil. 20, 04, 306-316.

Ansari A.M., Sylvester N.D., Shoham O., Brill J.P. (1990) A comprehensive mechanistic model for upward two-phase flow in wellbores, SPE Annual Technical Conference and Exhibition, Society of Petroleum Engineers.

Barnea D. (1986) Transition from annular flow and from dispersed bubble flow - Unified models for the whole range of pipe inclinations, Int. J. Multiph. Flow 12, 5, 733-744.

Du W. (2015) Research on technology of eddy current drainage gas production in West Sichuan Gas field. PhD Thesis, Southwest Petroleum University, China.

Facciolo L., Tillmark N., Talamelli A., Alfredsson P.H. (2007) A study of swirling turbulent pipe and jet flows, Phys. Fluids 19, 3, 035-105.

Fu J., Feng J., Chen P., Wei H., Liu Z. (2015) Simulation on wellbore pressure during dynamic kill drilling in deep water, Acta Pet. Sin. 36, 2, 232-237.

Hein N.W. (2007) A review of DOE testing of VortexFlow technology for Petroleum \& Natural Gas Production and Operations, in: Proceedings of the Annual Southwestern Petroleum Short Course, Southwestern Petroleum Short Course Association, TX, USA, 15 p.

Liu K., Sun L. (2017) Research on water drainage and gas recovery technology by vortex tool, China Pet. Mach. 45, 09, $87-91$.

Li K., Wang B., Hang Z., Ji H., Li H. (2017) Application of $\mathrm{K}$-means clustering in flow pattern identification of CCERT system, J. Beijing Univ. Aeronaut. Astronaut. 43, 11, 22802285.

Liu W., Bai B. (2015) Swirl decay in the gas-liquid two-phase swirling flow inside a circular straight pipe, Exp. Thermal Fluid Sci. 68, 187-195.

Milliken M. (2008) RMOTC-RMOTC tests Vortex Flow surface tools-Positive results were seen when units were put through tests dealing with pressure reduction and ice and paraffin blockage, World Oil, 229, 95-97.

Mingaleeva G.R. (2002) On the mechanism of a helical motion of fluids in regions of sharp path bending, Tech. Phys. Lett. 28, 8, 657-659.

Morsi S.A.J., Alexander A.J. (1972) An investigation of particle trajectories in two-phase flow systems, J. Fluid Mech. 55, 2, 193-208.

Petalas N., Aziz K. (1998) A mechanistic model for multiphase flow in pipes, Annual Technical Meeting, Petroleum Society of Canada.

Ryu S.H., Park G.C. (2011) A droplet entrainment model based on the force balance of an interfacial wave in two-phase annular flow, Nuclear Eng. Des. 241, 9, 3890-3897.

Shi Y., Yan T., Zhao X. (2018) Study on feasibility of the vortex tool for gas well fluid drainage in different flow regimes, China Pet. Mach. 46, 11, 116-120.

Singh K.A., Sarkar P., Praleya P., Sai G.S. (2016) Unconventional cyclone gas lift completion for offshore wells of Cambay Basin: A smart completion to optimize production and well intervention, SPE Annual Technical Conference and Exhibition, Society of Petroleum Engineers.

Surendra M., Falcone G., Teodoriu C. (2009) Investigation of swirl flows applied to the oil and gas industry, SPE Proj. Facil. Constr. 4, 01, 1-6.

Taitel Y., Dukler A.E. (1976) A model for predicting flow regime transitions in horizontal and near horizontal gas-liquid flow, AIChE J. 22, 1, 47-55.

Wei H., Zhi L., Wu W. (2014) Research development of unloading in gas well based on the swirling mechanism, Inner Mongolia Petrochem. Ind. 40, 07, 71-73.

Wu X., Zhou C., An Y., Liu X., Cen X. (2016) Dynamic analysis of liquid droplet and optimization of helical angle for vortex drainage gas recovery, Nat. Gas Ind. 36, 05, 45-50.

Yang T., Yu S., Yang H., Li J., Li N., Cao G., Wang Y. (2012) A new technology of vortex dewatering gas recovery in gas wells and its application, Nat. Gas Ind. 32, 08, 63-66.

Zhang C., Jin D., Wang J., Zhang J. (2012) Drainage gas recovery by downhole eddy current used for Sulige Gasfield, Nat. Gas Technol. Econ. 6, 05, 45-48.

Zhang Q., Li M. (2011) Theory and technology of oil production engineering, China University of Petroleum Press, Qingdao, pp. $40-75$.

Zhang Z., Liao R., Zhao Y., Cheng F., Liu J. (2018) Influence of guide vane number and arrangement pattern on performance of downhole vortex tools, J. Xi'an Shiyou Univ. (Nat. Sci. Ed.) 33, 05, 42-49.

Zhou C., Wu X., Zhang T., Zhao X. (2018) Experimental research for parameter optimization of the vortex tool for drainage gas recovery, Pet. Drill. Tech. 46, 06, 105-110.

Zhu Q., Zhang J., Xie F., Zeng C. (2013) Application of eddy drainage gas recovery to gasfields in Sichuan Basin, Nat. Gas Technol. Econ. 7, 01, 37-39. 